\title{
DESAFIOS DA MIGRAÇÃO DE PLATAFORMA DOS SISTEMAS DE OTIMIZAÇÃO DE PROCESSOS NA ARCELORMITTAL AÇOS PLANOS AMERICA DO SUL*
}

\author{
Arnaldo Veronez Junior ${ }^{1}$ \\ Lourival Silva Machado ${ }^{2}$ \\ Edilson José Machado Correa ${ }^{2}$
}

\section{Resumo}

Esse trabalho tem o objetivo de apresentar os principais desafios de natureza técnica e de gestão relacionados aos projetos de migração de sistemas baseados em plataformas descontinuadas ou com escassos recursos de suporte para outra mais atual, compatível com as novas tecnologias de aumento de produtividade e sem os riscos inerentes à obsolescência dos produtos empregados. Essas ações de migração de sistemas foram baseadas nas diretrizes estabelecidas no Plano Diretor de Tecnologia de Automação (PDTA), documento desenvolvido com o objetivo de orientar as ações do grupo de Engenharia de Automação no sentido de garantir a adoção de novas tecnologias de forma segura e planejada.

Palavras-chave: Automação; Tecnologia; Desenvolvimento; Gestão.

\section{CHALLENGES FACED DURING THE PLATFORM MIGRATION OF OPTIMIZATION PROCESS SYSTEMS OF ARCELORMITTAL FLAT CARBON SOUTH AMERICA}

\section{Abstract}

This work has the objective to present the main Technical and Management challenges related to migration projects that currently are based on discontinued platforms or with scarce resources support, by others systems with more recent technology, and able to use new technologies to Increase development productivity and without risks of obsolescence of product applied in it. These actions of migration systems were based on guidelines in the of Automation Technology Master Plan (PDTA) , which was created with objective of to guide the Automation Engineering group to ensure the adoption of new technologies in a safely and planned way.

Keywords: Automation; Technology; Development; Management.

1 Especialista em Engenharia de Produção, Especialista em Desenvolvimento de Automação e Instrumentação, ArcelorMittal Tubarão, Vitória, ES, Brasil.

2 Engenheiro Eletricista, Especialista em Desenvolvimento de Automação e Instrumentação, ArcelorMittal Tubarão, Vitória, ES, Brasil. 


\section{INTRODUÇÃO}

No ano de 2010 o grupo de automação de nível 2 da ArcelorMittal Tubarão concluiu um trabalho de definição de diretrizes de desenvolvimento e implantação de sistemas de automação de nível 2, chamado Plano Diretor de Tecnologia de Automação (PDTA). O resultado desse estudo apontou para diversas soluções tecnológicas que tem norteado os novos projetos da área nos últimos anos. Motivado principalmente pela mitigação de riscos relativos a ativos de automação, o PDTA indicou soluções como o emprego de sistemas virtualizados, centralização de bancos de dados, e criação de uma estrutura de software replicável a todos os sistemas de nível 2, chamado genericamente de "FRAMEWORK de nível 2", entre outros.

Desde então, diversos investimentos têm sido feitos na implantação da infraestrutura necessária à migração de sistemas para uma plataforma mais atual, baseada em virtualização de servidores e de terminais de operação, acesso a Bancos de Dados hospedados em estruturas de alta disponibilidade e compartilhado por diversas aplicações, e também na aplicação de um Framework capaz de otimizar o uso das melhores características das duas outras anteriormente citadas.

A seguir são apresentados os desdobramentos do trabalho do PDTA, em suas principais frentes:

\subsection{Implementação da Infraestrutura de Virtualização}

A tecnologia de virtualização de servidores já vem sendo usada em larga escala no mundo da TI, mas o seu emprego em TA ainda era bastante limitado. Essa solução, no entanto encaixou-se muito apropriadamente nas expectativas de se obter uma alternativa de alta confiabilidade para substituição do hardware baseado em plataformas Alpha Server que vinham sendo empregados tradicionalmente nos sistemas de nível 2 da AMT.

Com esse objetivo, implantou-se uma infraestrutura de virtualização composta por dois clusters, um na área da Aciaria e outro na área de utilidades, e essa infraestrutura vem sustentando a implementação de diversos novos sistemas e principalmente as migrações dos sistemas legados responsáveis pela otimização dos processos produtivos de diversas áreas operacionais.

\subsection{Implementação do Banco de Dados Centralizado}

Visando a maximização do uso de recursos computacionais, mais notadamente a aplicação de licenças de SGBD, optou-se por construir uma infraestrutura robusta com redundância geográfica que abrigaria os bancos de dados dos novos sistemas resultantes das migrações dos sistemas legados. Essa infraestrutura trouxe vários ganhos do ponto de vista de robustez e gestão do ambiente de banco de dados que antes não existia devido à solução baseada na instalação dos bancos de dados em cada servidor de aplicação individualmente.

A estrutura é baseada na solução Data Guard da Oracle e contempla a implementação de dois sites compostos cada um por servidor de Banco de Dados e storage, funcionando em regime de replicação contínua de dados entre sites de forma a garantir a integridade do banco em caso de falha de um dos sites. $O$ processo de chaveamento entre sites é feito manualmente, mas as aplicações não precisam de intervenção para passar a acessar o banco ativo. 


\subsection{Implementação do Framework de Nível 2}

Esse é o elemento central de todo o processo de migração de sistemas legados para uma nova plataforma mais moderna criada com o objetivo de disponibilizar um núcleo básico de software replicável para todos os futuros sistemas de nível 2.

Ele foi desenvolvido utilizando os conceitos mais atuais de orientação a objeto em linguagem java, e fazendo uso de produtos de mercado sempre que eles se mostravam aderentes aos requisitos das aplicações de automação de processos e possuíam comprovadas características de robustez técnica e com fornecedores de solidez comercial e de mercado.

O resultado dessa iniciativa representa um produto de total domínio da AMT, excetuando evidentemente os softwares de terceiros citados anteriormente, com capacidade de evolução, expansão e adaptação a novos requisitos emergentes.

\subsection{Planejamento das Migrações}

O conjunto dessas três iniciativas permitiu o progresso das atividades de migração que foram baseadas em um plano de investimentos estabelecido em etapas nas quais seria realizado um número determinado de projetos de migração, com base na capacidade orçamentária e na disponibilidade de recursos na época da sua realização.

Esse plano, em sua primeira etapa, que é o escopo desse trabalho, envolveu a realização de 6 projetos principais de migração. Durante a realização desses projetos surgiram ainda outros 3 subprojetos que contribuíram para a implementação técnica mais apropriada, conforme será abordado ao longo do texto.

\section{RESULTADOS E DISCUSSÃO}

Os próximos capítulos desse trabalho apresentam os principais desafios encontrados até o momento na tarefa de migrar os sistemas legados, focado principalmente no emprego da estrutura do Framework de nível 2 em projetos de migração de sistemas existentes que possuíam alto risco de paradas causadas por obsolescência de hardware e software.

\subsection{Desafios Tecnológicos}

\subsubsection{Servidor de banco de dados centralizado}

Durante o desenvolvimento dos projetos de migração, notou-se que a alternativa inicial de um Banco de Dados Centralizado para todos os sistemas, apesar de baseado em uma arquitetura robusta e de alta confiabilidade, deixava a desejar quando se partia para instalar nessa estrutura, Bancos de Dados de áreas diversas que possuíam diferentes requisitos, principalmente aqueles ligados a aspectos de paradas programadas, eventualmente necessárias para intervenções de manutenção nos Bancos de Dados, como por exemplo, a aplicação de patchs de correção ou atualizações de versão do SGBD. Essas diferenças de requisitos se tornaram tão fortes que forçaram a adoção de alternativa de centralização de Bancos de Dados de menor abrangência, mas que por outro lado trazia benefícios claros no que se refere à administração das situações anteriormente mencionadas.

A solução, no entanto, precisava continuar preservando os benefícios de economia de licenças, ao mesmo tempo em que garantisse uma estrutura de alta 
confiabilidade. Nesse sentido foi adotada uma solução baseada na estrutura de RAC (Real Application Cluster) da Oracle, com a adoção de servidores em cluster e chaveamento automático em caso de falha. A figura 1 ilustra a configuração da instalação aplicada à área da Aciaria.

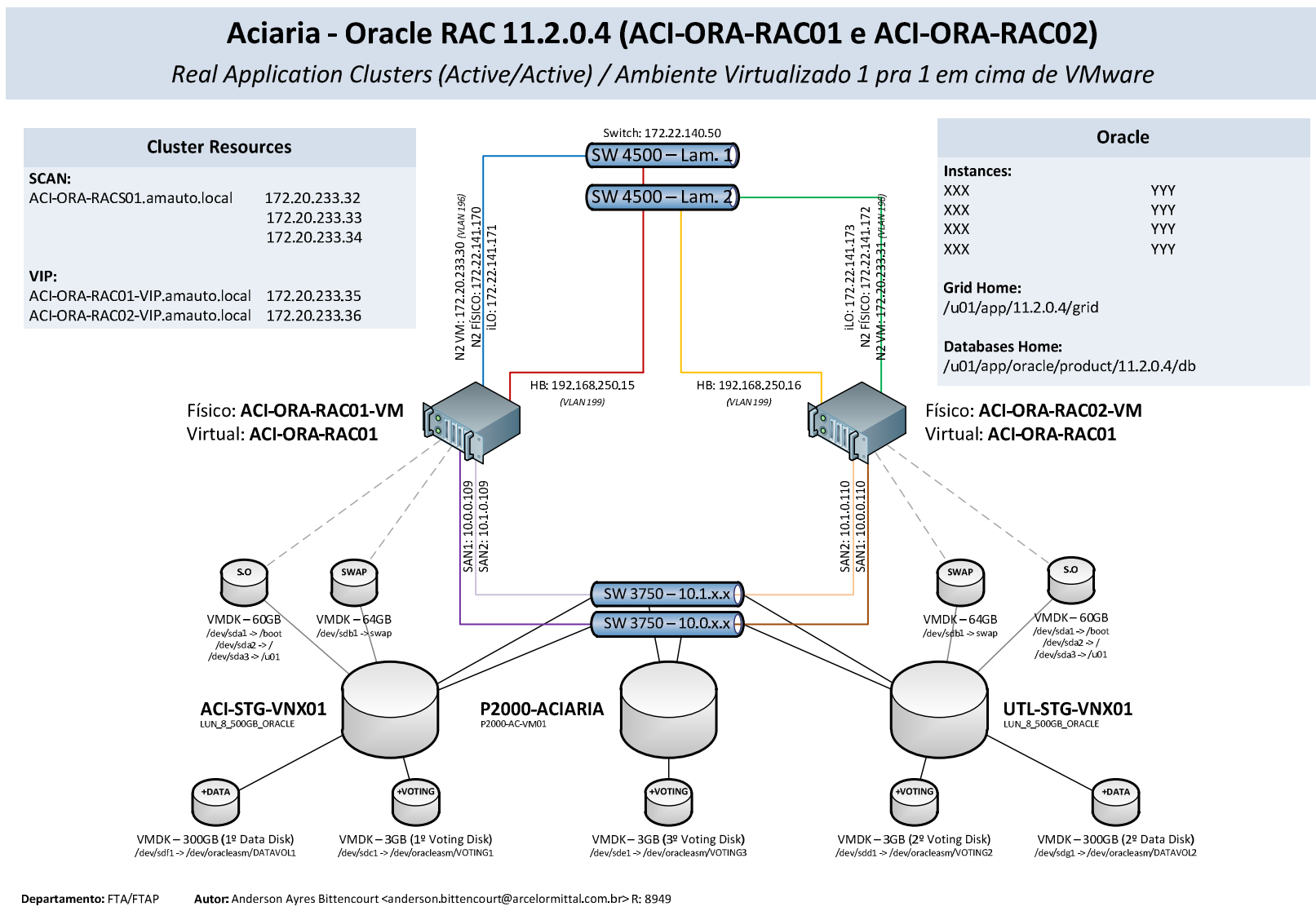

Figura 1. Arquitetura do Banco de Dados Centralizado da área da Aciaria.

Estruturas semelhantes a essa foram replicadas para as áreas de Redução e LTQ, de forma a abrigarem os bancos de dados dessas respectivas áreas.

\subsubsection{Servidor OMQ centralizado}

Os sistemas legados cresceram de forma não planejada e com isso a ferramenta usada para troca de mensagens entre eles não estava adequadamente estruturada, sendo que cada sistema abrigava seu próprio servidor de mensageria funcionando tanto como servidor quanto cliente. Essa arquitetura, com o passar do tempo e em consequência de alterações de condições comerciais, tornou-se inviável economicamente principalmente quando associada à solução de virtualização de servidores.

Para contornar essa situação optou-se por criar uma estrutura que centralizasse a distribuição das mensagens de determinadas áreas, como Aciaria, Redução e LTQ. Essas três estruturas, apresentadas na figura 2, foram criadas de forma semelhante entre si e se baseiam em servidores em cluster com alta disponibilidade, o que permite o uso de licenças cliente do software de mensageria nos servidores de aplicação, propiciando maior economia, pois o software cliente não tem custo. Tecnicamente a solução não traz nenhum inconveniente e permite uma administração mais simplificada. 


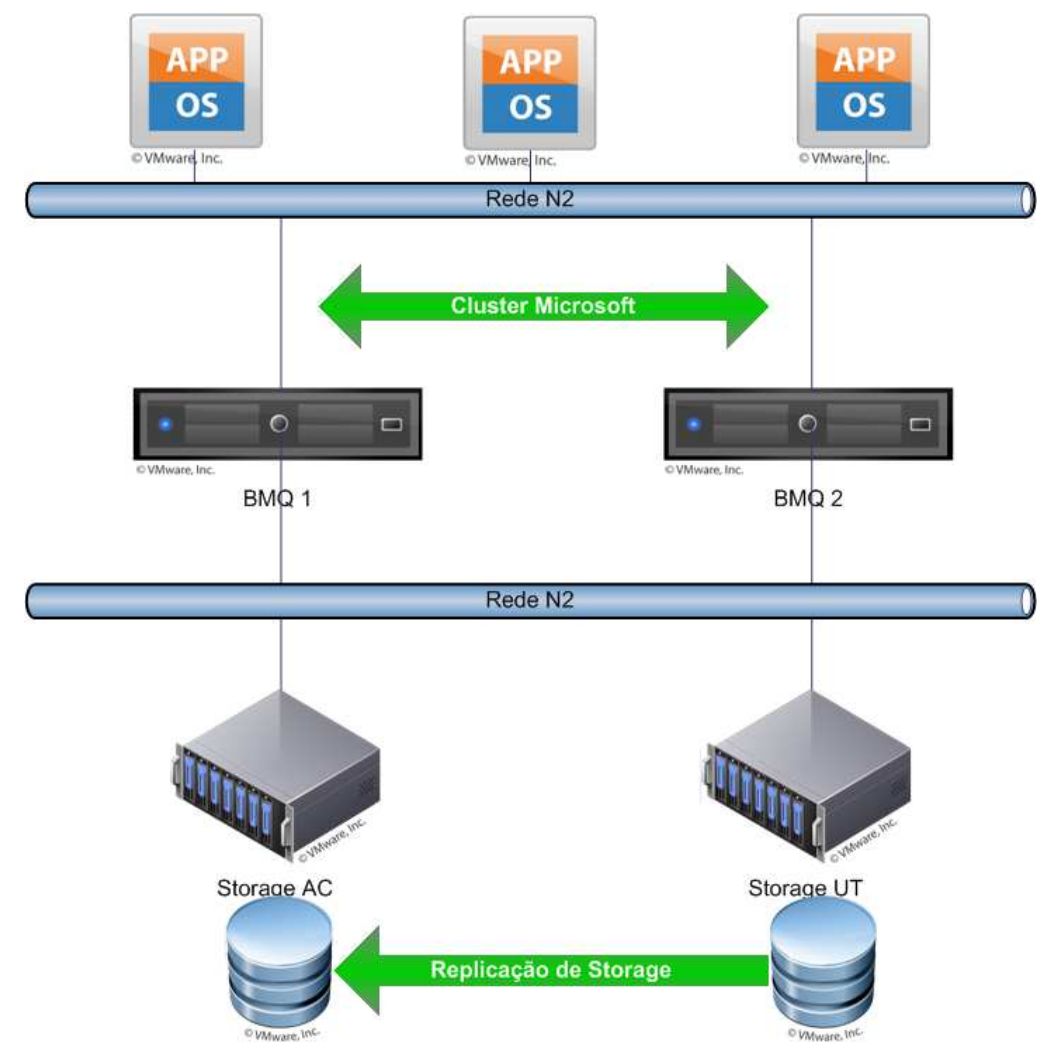

Figura 2. Arquitetura do Sistema Centralizado de Mensageria.

\subsubsection{OPC UA}

A comunicação com o chão de fábrica sempre foi uma das maiores preocupações nos projetos de migração de sistemas. A definição pela comunicação OPC foi tomada nas primeiras etapas do PDTA, porém a sua restrição à plataforma Windows indicava claramente que essa não deveria ser a solução definitiva para essa questão já que a convivência do Java com o protocolo OPC sempre foi dificultada por questões de compatibilidade dos componentes DCOM essenciais à comunicação OPC DA. Durante o desenvolvimento das aplicações na nova plataforma, foram adotadas diferentes soluções para compatibilização, porém nenhuma delas permitiu que a questão de comunicação com os sistemas de controle das plantas fosse adequadamente resolvida no ambiente de produção. Por outro lado, a expectativa que o protocolo OPC UA se tornasse efetivo no ambiente de automação de processos, manteve a atenção da equipe sempre voltada para a aplicação dessa tecnologia, o que permitiria a independência da plataforma conforme era a nossa intenção inicial.

A união dessas duas situações, fragilidade da comunicação OPC DA e a busca pela alternativa de comunicação OPC UA levou à necessidade de testar diversos produtos que viabilizassem o uso dessa tecnologia. Os testes executados foram bem sucedidos e essa opção foi definitivamente adotada para os sistemas com implantação mais recente. Com isso, foi possível alcançar não só o objetivo de ter estabilidade e confiabilidade na coleta de dados do processo como também atingimos a nossa intenção inicial de independência da plataforma Windows. 


\subsection{Desafios de Gestão}

\section{Gestão do Framework}

A adoção de um produto totalmente customizado trouxe uma série de benefícios, entre eles o domínio técnico por parte da equipe de especialistas da Engenharia de Automação de todos os componentes, sejam documentos ou códigos fonte, permitindo dispor deles como melhor convier, seja alterando, cancelando, substituindo total ou parcialmente ou incluindo novas partes ao produto original. No entanto, essa autonomia implicou na necessidade de uma visão diferenciada sobre a questão da administração desse produto.

Com esse intuito, foi criado um grupo de especialistas que compõem o "Comitê Técnico do Framework" que é responsável pela condução das necessidades de melhorias e correções a serem aplicadas ao produto, bem como o controle de versionamento. Esse grupo analisa as demandas por melhorias e correções e decide a sua pertinência, abrangência, impacto e esforço para determinar quais delas deverão ser tratadas com prioridade e quais poderão ser tratadas em um momento posterior.

Lições Aprendidas no Desenvolvimento de Projetos

Com o desenvolvimento e a implantação dos sistemas baseados no Framework, diversos aprendizados foram identificados e estão sendo tratados como "Lições Aprendidas". Basicamente, a partir de projetos realizados, será construída uma base de dados de lições aprendidas que virá a ser fonte de informação para futuros projetos, através de uma metodologia que controle a geração/consumo das informações.

Dentre os benefícios esperados com o uso dessa metodologia nos projetos pode-se citar:

- Sistematização no planejamento e execução de projetos;

- Identificação e mitigação de riscos;

- Redução de perdas;

- Ganho de produtividade;

- Identificação de pontos fortes, fracos e ações para evolução;

\subsection{Desafios de Recursos}

Um dos pontos de atenção no plano de migrações foi e tem sido a questão de recursos humanos capacitados para o uso das ferramentas tecnológicas envolvidas nesse processo. Intencionalmente, a equipe de Automação da ArcelorMittal é um tanto quanto eclética na sua formação. Isso permite a formação de equipes de projeto com sinergia entre os seus membros, porém traz a necessidade de capacitações complementares nas novas ferramentas tecnológicas adotadas. Assim, para a consecução do plano de migrações de sistemas legados, foi elaborado e executado um plano de capacitação das equipes nas novas tecnologias.

No mesmo sentido, considerou-se importante a busca e/ou o desenvolvimento de empresas parceiras, com expertise nas novas tecnologias para atendimento às necessidades dos projetos e manutenções e melhorias futuras nestes sistemas.

\subsection{Evolução}

Como um produto que deve ser sempre mantido atualizado e otimizado para que possa atender adequadamente as demandas dos projetos de migração, o 
Framework de nível 2 da automação tem um horizonte de agregar novas características funcionais que são brevemente descritas abaixo:

Adaptação para aplicações com características de TI:

Diversos sistemas existentes que são responsabilidade da área de automação, são simplesmente aplicações de consulta a banco de dados para geração de relatórios gerenciais de complexidade variada. A aplicação do Framework de sistemas de automação de nível 2 nesses casos, implica na inutilização de diversos módulos funcionais nativos do framework. A ideia dessa melhoria é criar um ambiente específico para sistemas desse tipo para tornar essas aplicações mais eficientes e compatíveis com as tecnologias empregadas no framework.

Adaptação para aplicações de controle:

$\mathrm{Na}$ forma atual, o framework possui limitações para aplicações em sistemas de controle que requeiram alta velocidade de processamento e aquisição de dados de processo. Essas limitações são basicamente ligadas ao tempo de coleta de dados que é de certa forma limitada pelo protocolo OPC a frequências máximas na faixa de 1 segundo. A intenção dessa adaptação é tornar o sistema capaz de atender a sistemas com requisitos mais exigentes de velocidade de processamento.

Novo sistema de mensageria

Até o momento a integração de novos sistemas Framework tem sido baseada em uma solução de mensageria cara, usada em sistemas legados, que não teve a evolução técnica desejada e que está descontinuada. Para o futuro, será analisada uma evolução para um sistema de mensageria robusto e atualizado para substituir o existente.

- Portabilidade do Framework

Um dos motivos da opção pela plataforma Java foi a expectativa de portabilidade da aplicação para outros sistemas operacionais. No entanto, algumas escolhas feitas em decorrência de necessidades dos projetos, limitaram de certa forma essa característica desejável. O objetivo dessa melhoria será corrigir esses desvios e tornar essa aplicação realmente portável de forma a disponibilizar alternativas mais baratas de sistemas operacionais.

- Aumento de produtividade

Uma das expectativas para a plataforma de Framework de Automação é a possibilidade de aumento de produtividade no desenvolvimento de sistemas. Nesse sentido, uma evolução esperada no Framework é a implementação de ferramentas e mecanismos que permitam o aumento da produtividade no desenvolvimento de novos sistemas.

\section{CONCLUSÃO}

Finalmente, podemos concluir que as atividades relativas à migração de sistemas legados para a nova plataforma baseada no Framework de nível 2 da ArcelorMittal Tubarão representou um verdadeiro desafio para a equipe de automação, que teve que conviver com um momento de profundas mudanças e de situações inéditas provocadas por inovações significativas em todos os campos relacionados ao desenvolvimento de sistemas de nível 2, desde aspectos puramente tecnológicos ou de gestão, passando por necessidades maciças de capacitação de pessoal até a administração dos recursos financeiros para as diversas frentes de projeto.

Como resultado final ficou uma rica coleção de lições aprendidas que certamente irá contribuir muito para o sucesso dos próximos projetos, bem como um aperfeiçoamento nos processos de gestão de projetos com consequente 
aculturamento das equipes envolvidas nas questões de desenvolvimento de projetos.

\section{BIBLIOGRAFIA}

1 Corrêa, E.J.M. Plano Diretor de Tecnologia de Automação da Arcelormittal Tubarão, 2012, Belo Horizonte - MG.

2 Pedrini,D.C., Corrêa, E.J.M.,Nunes,L.S. Aplicação de Virtualização de Servidores em Sistemas de Automação da Arcelormittal Tubarão, 2012, Belo Horizonte, MG. 